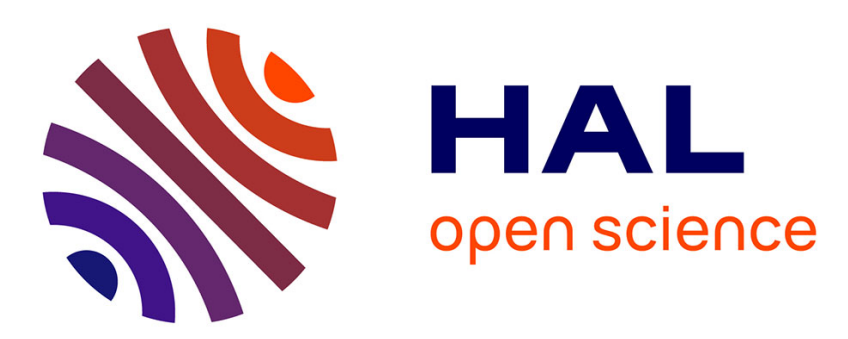

\title{
Geometry of dislocations in icosahedral quasicrystals
}

Denis Gratias, Jean-Tristan Beauchesne, Frédéric Mompiou, Daniel Caillard

\section{To cite this version:}

Denis Gratias, Jean-Tristan Beauchesne, Frédéric Mompiou, Daniel Caillard. Geometry of dislocations in icosahedral quasicrystals. Philosophical Magazine, 2006, 86 (25-26), pp.4139-4151. 10.1080/14786430600575435 . hal-00513660

\section{HAL Id: hal-00513660 \\ https://hal.science/hal-00513660}

Submitted on 1 Sep 2010

HAL is a multi-disciplinary open access archive for the deposit and dissemination of scientific research documents, whether they are published or not. The documents may come from teaching and research institutions in France or abroad, or from public or private research centers.
L'archive ouverte pluridisciplinaire $\mathbf{H A L}$, est destinée au dépôt et à la diffusion de documents scientifiques de niveau recherche, publiés ou non, émanant des établissements d'enseignement et de recherche français ou étrangers, des laboratoires publics ou privés. 


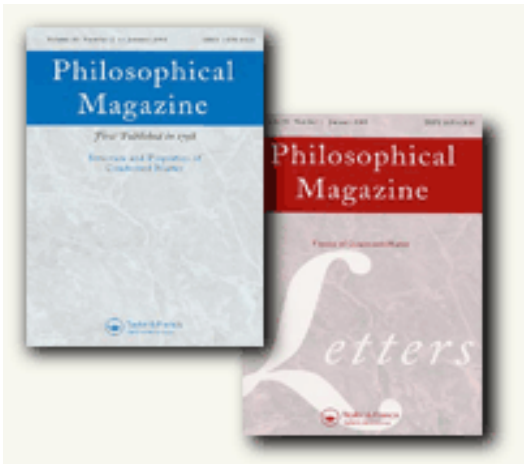

\section{Geometry of dislocations in icosahedral quasicrystals}

\begin{tabular}{|r|l|}
\hline Journal: & Philosophical Magazine \& Philosophical Magazine Letters \\
\hline Manuscript ID: & TPHM-05-Oct-0459.R1 \\
\hline Journal Selection: & Philosophical Magazine \\
\hline Author: & 06-Jan-2006 \\
\hline Complete List of Authors: & $\begin{array}{l}\text { Gratias, Denis; CNRS-ONERA, LEM } \\
\text { Beauchesne, Jean-Tristan; ONERA, LEM } \\
\text { Mompiou, Frederic; NIST, Metallurgy } \\
\text { Caillard, Daniel; CEMES - CNRS, Laboratoire d Optique Electronique }\end{array}$ \\
\hline Keywords: & quasicrystalline alloys, dislocation theory \\
\hline Keywords (user supplied): & quasicrystal, dislocation, plasticity \\
\hline \multicolumn{2}{|l|}{$\begin{array}{l}\text { Note: The following files were submitted by the author for peer review, but cannot be converted } \\
\text { to PDF. You must view these files (e.g. movies) online. }\end{array}$} \\
\hline DGratiasetal.tex \\
\hline
\end{tabular}

\section{今) ScholaroNE" Manuscript Central}




1
2
3
4
5
6
7
8
9
10
11
12
13
14
15
16
17
18
19
20
21
22
23
24
25
26
27
28
29
30
31
32
33
34
35
36
37
38
39
40
41
42
43
44
45
46
47
50
58
59
50
52
53
50

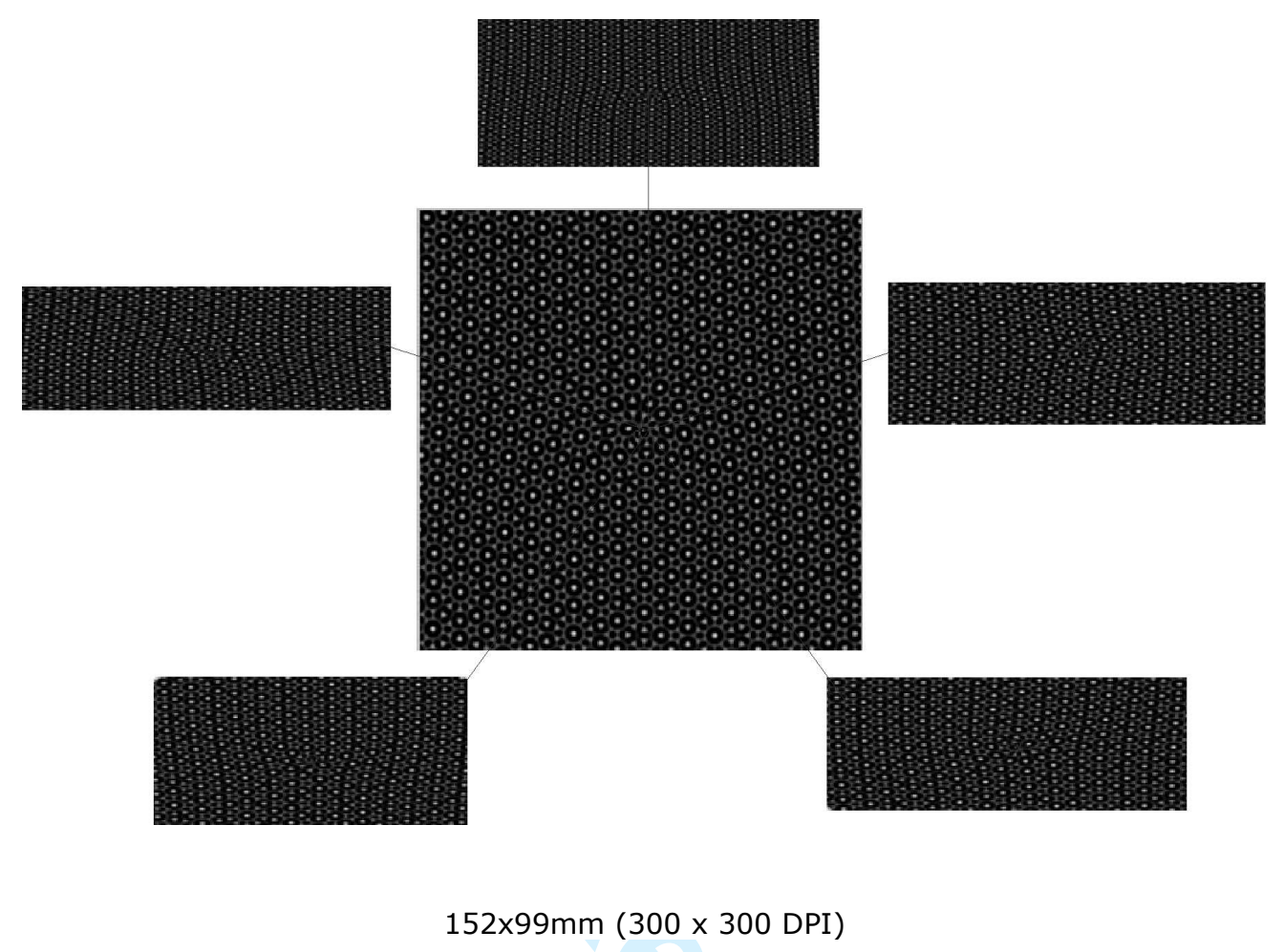

http://mc.manuscriptcentral.com/pm-pml 

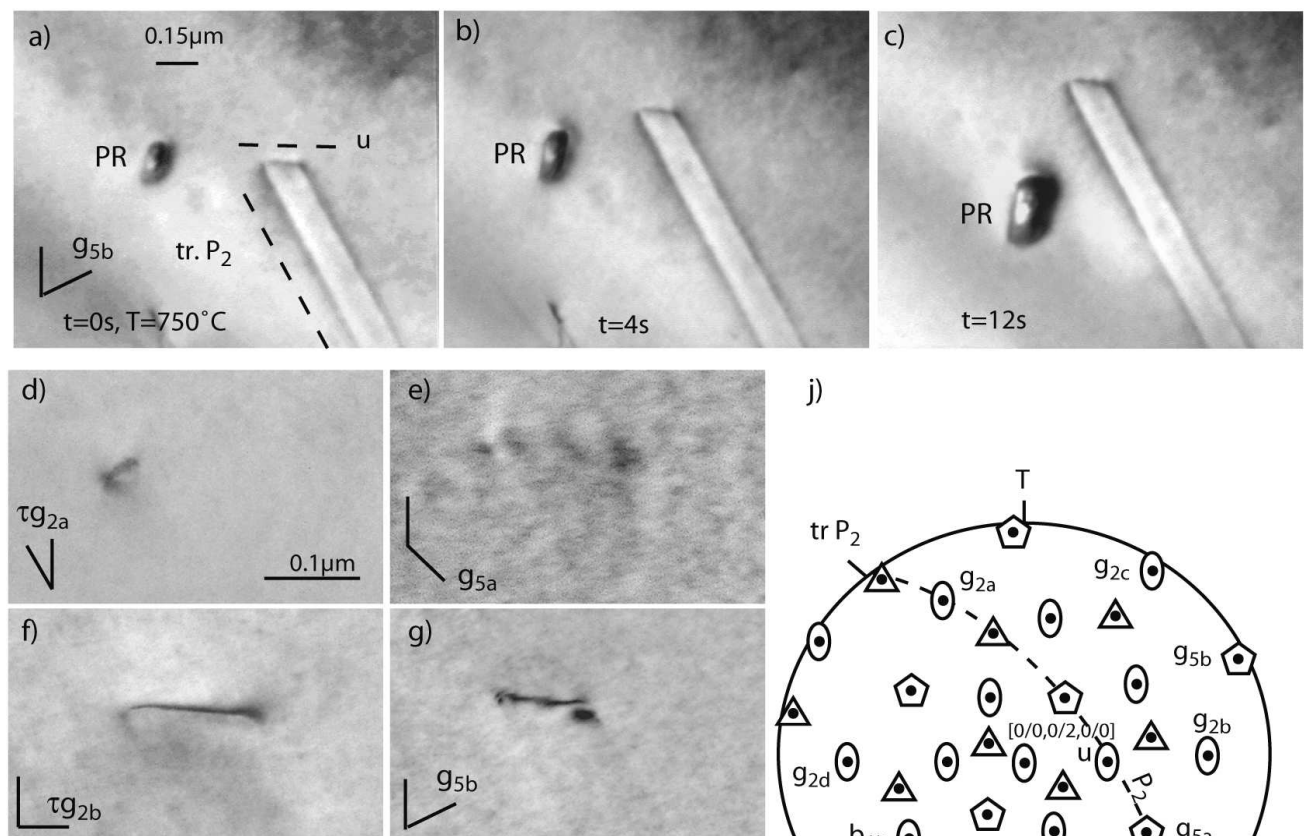

j)
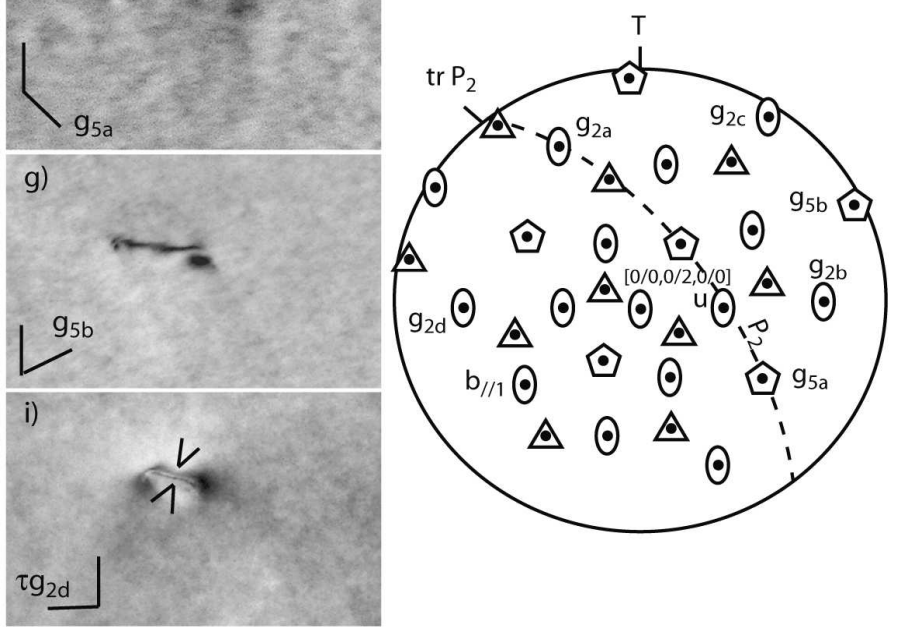

$141 \times 116 \mathrm{~mm}(300 \times 300 \mathrm{DPI})$ 


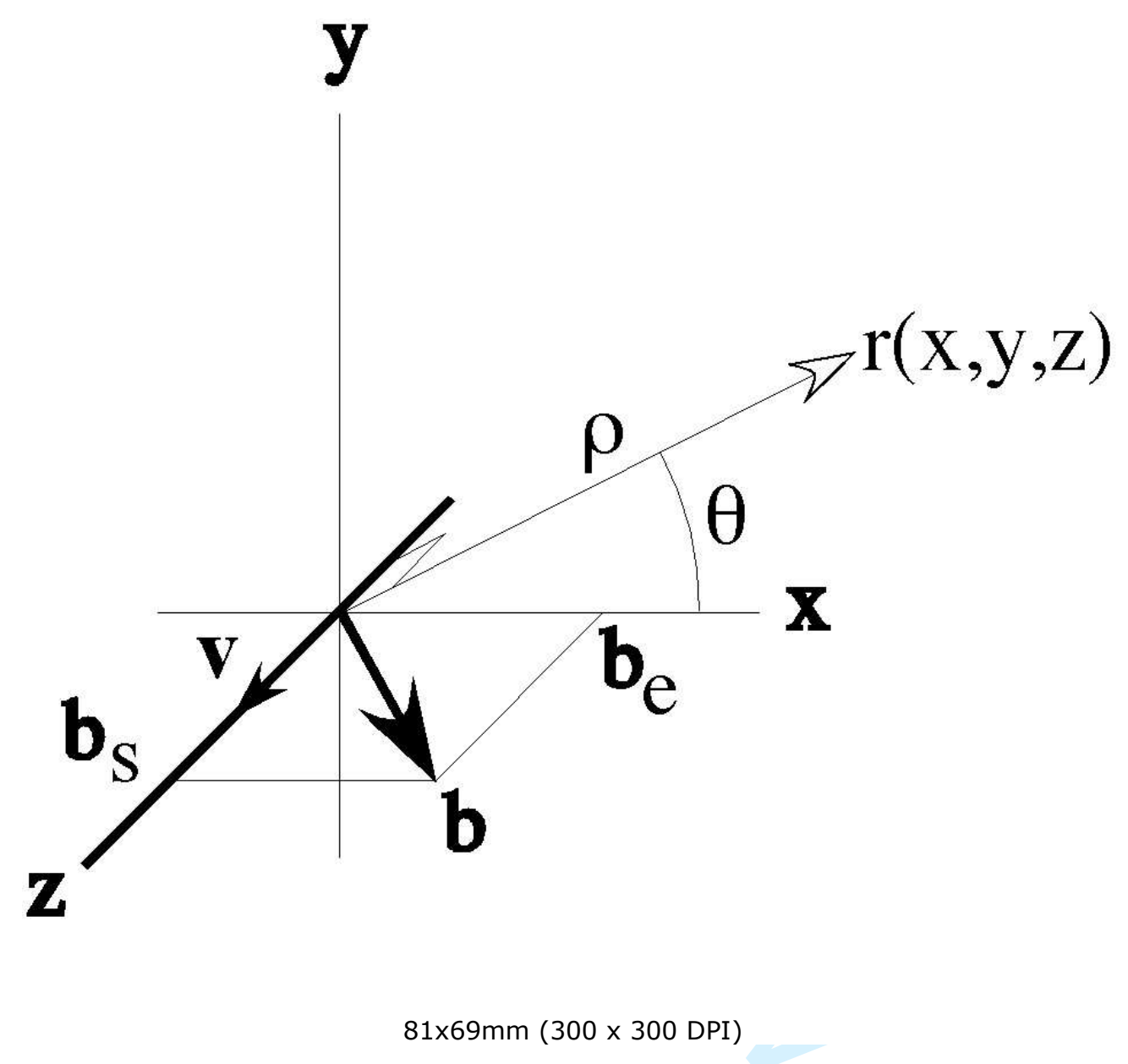




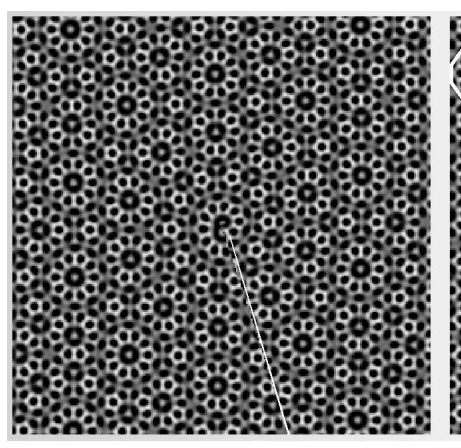

(a)

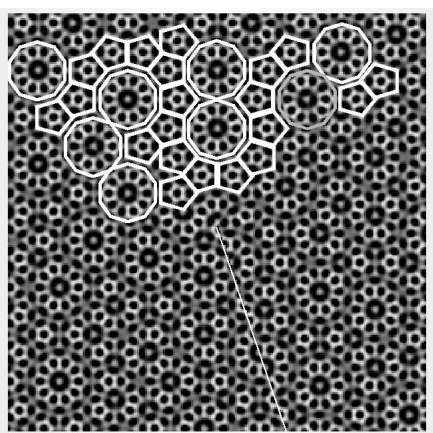

(b)

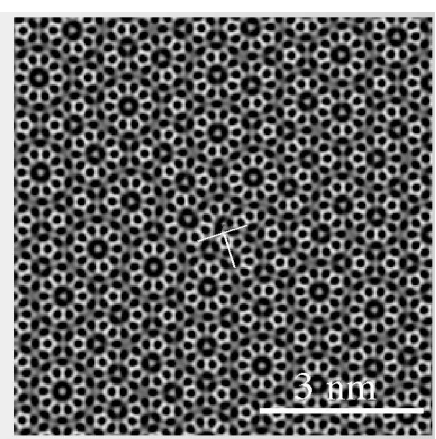

(c)

$165 \times 64 \mathrm{~mm}(300 \times 300 \mathrm{DPI})$ 

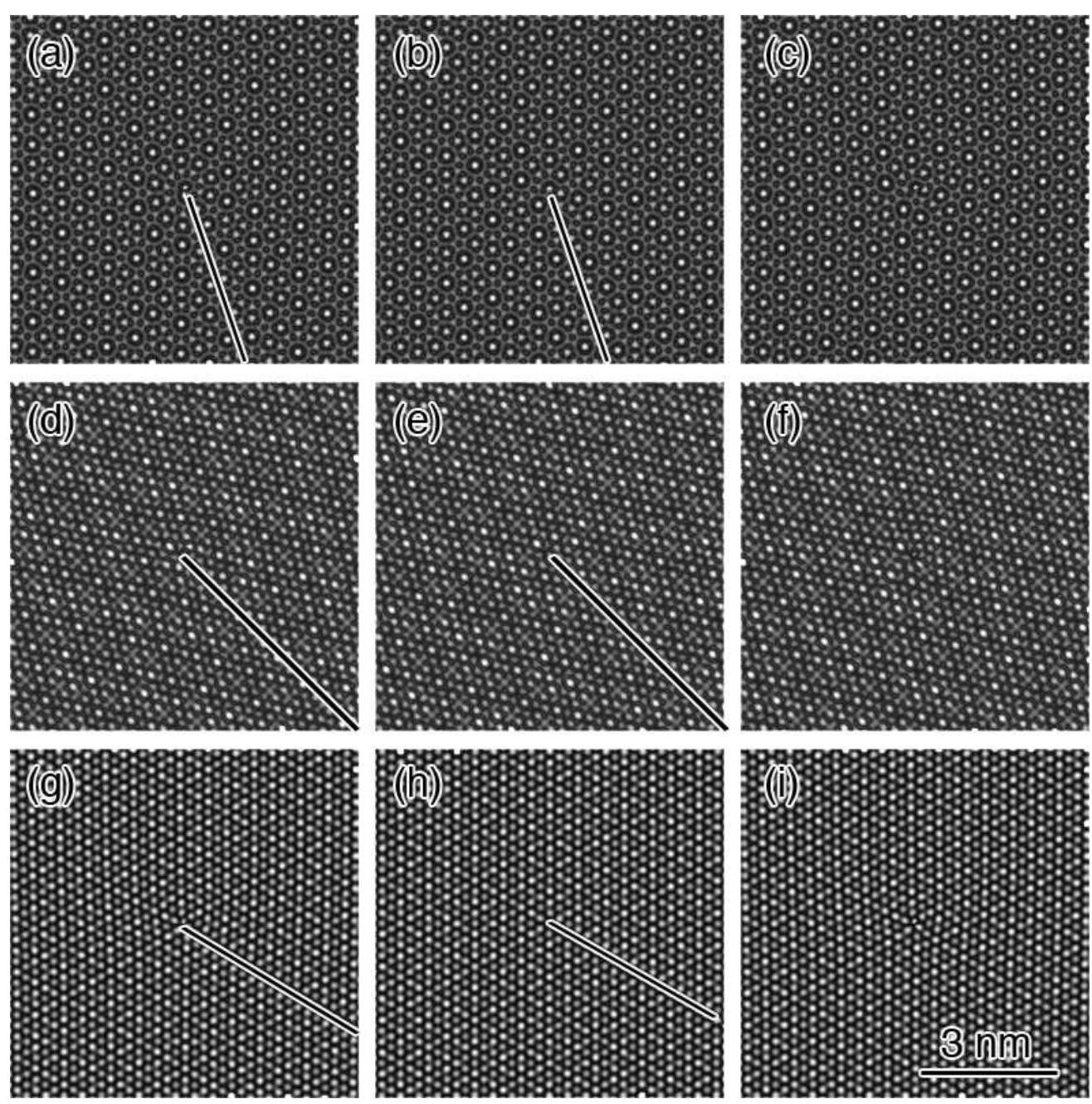

$279 \times 280 \mathrm{~mm}(72 \times 72 \mathrm{DPI})$ 


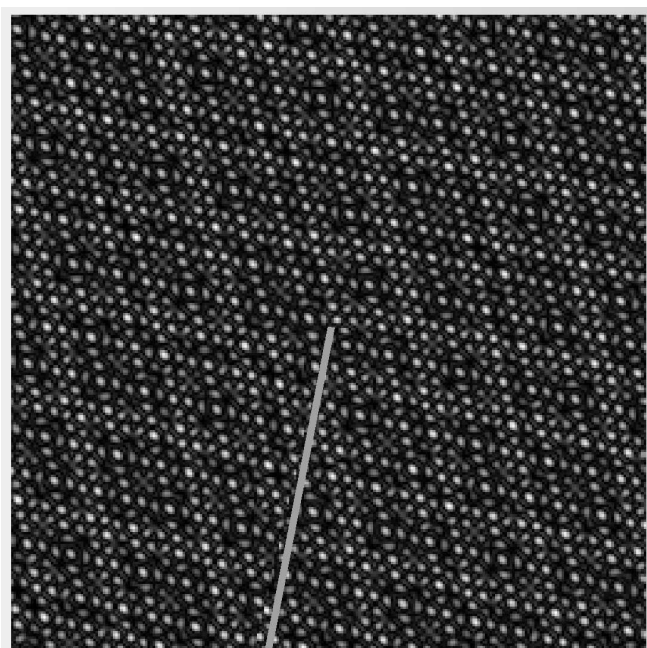

$(-1,1,1,1,1,-1) / 2$

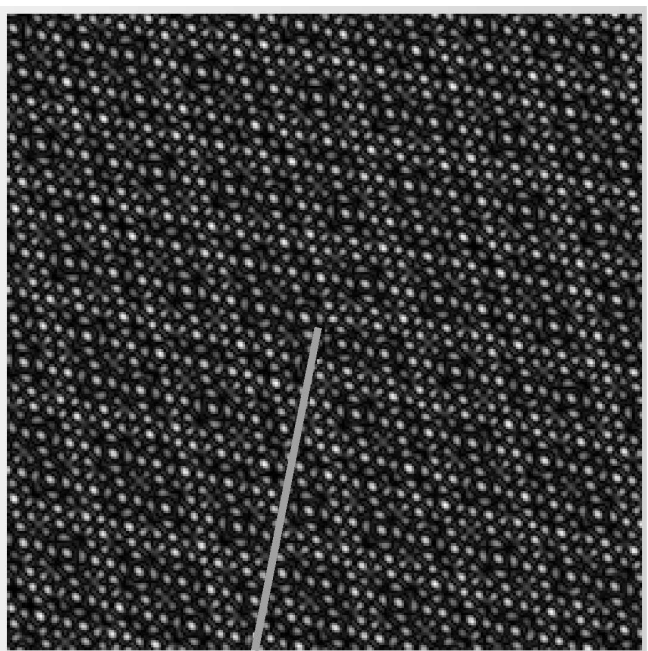

$(1,0,0,0,0,0)$

$184 \times 99 m m(300 \times 300$ DPI $)$ 


\title{
Geometry of dislocations in icosahedral quasicrystals
}

Denis Gratias, Jean-Tristan Beauchesne, LEM-CNRS/ONERA, B.P. 72

92322 Châtillon cedex, France

Frédéric Mompiou, Metallurgy Division, NIST, 100 Bureau Dr. stop 8555

Gaithersburg 20899 MD, USA and Daniel Caillard CEMES-CNRS, 29, rue Jeanne Marvig B.P. 4347 31055 Toulouse cedex 4, France

\begin{abstract}
Quasicrystals are complex metallic alloys that are ductile at high temperatures close to their melting point. It has been early recognized that their plasticity is due to a flux of moving linear defects in all respects similar to dislocations in ordinary crystals. The present paper is an attempt to propose in a short didactic review a simple synthetic analysis of the basic underlying geometry of quasicrystalline dislocations exemplified with experimental and calculated images of electron microscopy in icosahedral phases.
\end{abstract}

\section{Introduction}

This paper is a short contribution to the analysis of the geometrical properties of dislocations in icosahedral quasicrystals [1, 2]. Its main goal is to present, as a short review, a detailed description of these defects in the framework of $N$-dimensional crystallography.

It has been early recognized that, like many other intermetallic compounds, icosahedral quasicrystals are very brittle at low temperature but exhibit a unusually large plastic regime at high temperatures 
which is essentially due to the motion of linear defects in all respects similar to ordinary dislocations (see for instance $[3,4]$ ).

From a geometrical point of view, these original deformation modes are qualified as dislocations $[5,6,7,8]$ because by its very definition, a (icosahedral) quasicrystal can be described as the $3 D$ cut along an irrational orientation of an abstract $6 D$-periodic object $[9,10,11,12]$. As periodicity is recovered in this abstract $6 D$ space, the notion of dislocation is a natural extension of the one classically defined for crystals. This abstract $6 D$ space decomposes into two complementary subspaces :

- the $3 D$ physical space that we shall call the parallel space and note $\mathrm{E}_{\|}$;

- the perpendicular space, also of dimension 3, that we note $\mathrm{E}_{\perp}$.

Quasiperiodic structures present in real space atomic planes and rows, exactly like ordinary crystals with the only difference that the constitutive atoms are not periodically but quasiperiodically spaced (see for instance [9]). An important theorem [13] states that for the case where the $6 D$ lattice nodes project onto $\mathrm{E}_{\perp}$ as an uniformly dense set of points - as it is the case of the icosahedral phase - any two parallel cuts along $\mathrm{E}_{\|}$generate locally isomorphic quasicrystalline structures that are physically equivalent to any finite distance, $i$. e. any two such quasicrystals are physically indistinguishable in all respects. This basic gauge invariance corresponds to the fact that the physical properties of quasicrystalline dislocations must be independent of the choice of origin in $\mathrm{E}_{\perp}$ during the cut process generating the real atomic structure. As we shall see next, this invaraince induces important geometric constraints in the description of quasicrystalline dislocations.

\section{Geometry of quasicrystalline dislo- cations}

\subsection{Burgers vector}

The definition of a dislocation in a (Euclidean) $N$-dimensional $(N D)$ medium follows the Volterra process used for usual $3 D$ media : a cut is performed along a $(N-1) D$ oriented (hyper)surface $\Sigma$ bounded by a closed $(N-2) D$ (hyper) line; the upper part of the medium at the cut level is displaced by a finite vector $\vec{B}$, with respect to the lower part. The medium is finally re-glued and mechanically relaxed. 
If $\vec{B}$ is a translation belonging to the translation invariance group of the medium, the $\Sigma$ cut surface vanishes and an elastic singularity is created at the level of the (hyper)line that we call a perfect dislocation with Burgers vector $\vec{B}$. For the case of a $N D$-periodic object with hyperlattice $\Lambda$, the Burgers vector $\vec{B}$ of a perfect dislocation is a hyperlattice translation : $\vec{B} \in \Lambda$ and therefore it has necessarily one non zero components in each $\mathrm{E}_{\|}$and $\mathrm{E}_{\perp}$ subspaces:

$$
\vec{B}=\vec{b}_{\|}+\vec{b}_{\perp} \quad \in \Lambda
$$

An example of a (computed) dislocation, seen end on, of Burgers vector $B=(0,0,1,0,0,-1)(6 D$ notation from the indexing scheme proposed by Cahn et al. [14]) in an icosahedral phase is presented on Fig. 1. The observation plane is perpendicular to a 5-fold direction. As for ordinary dislocations, observation at glancing angle along certain directions shows kinds of additional half-planes the number and stacking of which depends on the chosen direction.

\subsection{Dislocation line}

The dislocation line, noted $V$, is a $(N-2) D$-manifold. For the icosahedral case, the line $V$ is a $6-2=4 D$ manifold. Closed circuits can be constructed around $V$ in the remaining $2 D$-subspace, that will be used to define the Burgers vector. This means that Burgers circuits are entirely defined in $\mathrm{E}_{\|}$and are indeed pertinent physical quantities that can be experimentally determined ${ }^{1}$.

The invariance stating that the dislocation description is independent of the choice of the origin in $\mathrm{E}_{\perp}$ fixes the "orientation" of the dislocation line $V$ : the only generic way for $V$ to be invariant by any translation of the cut along $\mathrm{E}_{\perp}$, is that $V$ contains $\mathrm{E}_{\perp}$. The dislocation $4 D$-line decomposes therefore into a $3 D$-subspace parallel to $\mathrm{E}_{\perp}$ plus a remaining $1 D$-subspace that is necessarily in $\mathrm{E}_{\|}$: this is the usual real dislocation line $\vec{u}$ in physical space.

Thus a (perfect) dislocation in an icosahedral quasicrystal is made of a Burgers vector $\vec{B}$ belonging to the $6 D$-lattice of the quasicrystal and a dislocation line of dimension 4 that decomposes into a $1 D$ line in $\mathrm{E}_{\|}$and a $3 D$-subspace parallel to $\mathrm{E}_{\perp}$. It formally decomposes as $V=\vec{v}_{\|} \oplus \mathrm{E}_{\perp}$.

Since $\vec{B}$ has a non zero component in $\mathrm{E}_{\perp}$ it is stricto sensu, impossible to construct pure edge (perfect) dislocations in an icosahedral

\footnotetext{
${ }^{1}$ This does not imply $\vec{B}$ to lie in this $2 D$-subspace: in fact $\vec{B}$ is in a rational $2 D$-plane with respect to $\Lambda$ that is oblique with respect to $E_{\|}$. It is the irrationality of this oblique orientation that makes the relation between the lattice nodes of this rational plane and their projections in $\mathrm{E}_{\|}$a one-to-one correspondence.
} 
phase because of $\mathrm{E}_{\perp}$ being embedded in $V$. Hence, edge and screw dislocations are better defined in quasicrystals in considering only the relative orientation of the Burgers vector with respect to the real physical space irrespective of the components of the Burgers vector onto $\mathrm{E}_{\perp}$. We thus define the edge and screw components, respectively $\vec{b}_{e}$ and $\vec{b}_{s}$ of a dislocation by the usual scalar products $\vec{b}_{s}=\left(\vec{b}_{\|} \cdot \vec{u}_{\|}\right) \vec{u}_{\|}$, and $\vec{b}_{e}=\vec{b}_{\|}-\vec{b}_{s}$.

If the dislocation line $\vec{u}_{\|}$has a specific orientation along well defined quasicrystallographic directions (as it is often the case in crystals), the corresponding $4 D$ dislocation (straight) line $V$ contains a $2 D$ sublattice of $\Lambda$ the trace of which in $\mathrm{E}_{\|}$is the physical dislocation line $\vec{u}_{\|} \cdot$

\subsection{Displacement field induced by a quasicrys- talline dislocation}

As imposed by the decomposition (1) any dislocation in a quasicrystal generates two displacement fields of different physical nature.

The first one is related to the parallel component $\vec{b}_{\|}$of the $6 D$ Burgers vector $\vec{B}$ and is governed, in a good approximation, by standard elastic theory. Its stored elastic energy varies like $\left|b_{\|}\right|^{2}$ and the interaction forces between dislocations and stress fields can be computed using the usual tools of elasticity theory. This displacement field is referred to as the phonon field although - irrespective of a global dislocation motion - it describes static displacements of atoms.

The second displacement field is non trivial and is specific to quasicrystals and incommensurate structures. It is generated by the perpendicular component $\vec{b}_{\perp}$ of the $6 D$ Burger vector and extends in $\mathrm{E}_{\perp}$. It is not attached to any local elastic displacement of atoms but to a rearrangement of the local atomic configurations with respect to each others with no change in their shapes. It is closely related to the configurational energy of the alloy on both chemical and topological order and is depend on the thermodynamic aspects of the material. It is (improperly) called the phason field as a reference to the phason modes encountered in dynamical studies of incommensurate structures. The expression of the energy attached to this phason field is either proportional to $\left|b_{\perp}\right|$ or $\left|b_{\perp}\right|^{2}$ depending on which model (perfect tiling or random tiling) is chosen to explain the stability of the quasicrystal. So far, the most advanced study on the subject has been made on decagonal phases by Koschella [20] who concluded that the phason energy is best described by a quadratic term in phason fluctuations. In that scheme, the dislocation energy associated to the local tiling faults around the dislocation would vary as $\left|b_{\perp}\right|^{2}$. 
The global $6 D$-displacement field $\vec{U}$ induced by a dislocation decomposes on respectively $\mathrm{E}_{\|}$and $\mathrm{E}_{\perp}$ as $\vec{U}=\vec{u}_{\|}+\vec{u}_{\perp}$. The displacement field $\vec{U}(\vec{X})$ depends only on the parallel component, $x_{\|}$, of the running vector $\vec{X}$, so that:

$$
\vec{U}(\vec{X})=\vec{U}\left(x_{\|}\right)=\vec{u}_{\|}\left(x_{\|}\right)+\vec{u}_{\perp}\left(x_{\|}\right)
$$

as the dislocation "line" contains $\mathrm{E}_{\perp}$ so that the total $6 D$-displacement field depends only on the physical space variable. Integrated along a closed circuit $\mathcal{C}_{\|}$in $\mathrm{E}_{\|}$, these two fields lead to:

$$
\oint_{\mathcal{C}_{\|}} \overrightarrow{d u} \vec{u}_{\|} \vec{b}_{\|} \quad \oint_{\mathcal{C}_{\|}} \overrightarrow{d u_{\perp}}=\vec{b}_{\perp}
$$

if the circuit encloses the dislocation line $V$, and zero otherwise.

\subsection{Partial dislocations and dissociations}

The notion of partial dislocations in quasicrystals follows the one defined for crystals: a partial dislocation has a Burger vector that is an integer fraction of a lattice translation. Such dislocations are bounded by $2 D$ extended faults with fault vectors equal to the Burgers vector (up to a lattice translation of $\Lambda$ ). The $i$-AlPdMn F-type icosahedral phase has specific atomic structures where the atoms distribute on three main quasiperiodic networks displaced from each other by $(1,0,0,0,0,0)$ and $(\overline{1}, 1,1,1,1, \overline{1}) / 2$. These translations and their combinations can be called super partial dislocations in the sense that they belong to the $P(A)$-lattice for $(1,0,0,0,0,0)$ and $D_{x(y)}^{6}(A)$-lattice for $(\overline{1}, 1,1,1,1, \overline{1}) / 2$ which both are superlattices of rank 2 of the $F(2 A)$ lattice. These translations are good candidates for possibly generating antiphase boundaries (both with phase shifts 0 or $\pi$ in the $6 D$ space) in the material. So far only superpartial dislocations of Burgers vectors $(1,0,0,0,0,0)$ and $(1,0, \overline{1}, 0,0,1)$, both of the same family, have been observed in these phases [24].

Quasicrystals exhibit a specific type of dissociation that results from the very basic physical dissymmetry between the phonon and the phason spaces in the canonical decomposition $\vec{B}=\vec{b}_{\|}+\vec{b}_{\perp}$. A dislocation appears as a strip of fault bounded on the head, by the singularity $\vec{b}_{\|}$, and on the tail, by $\vec{b}_{\perp}$. All the elastic distortion is concentrated on the head line whereas the tail line corresponds to the termination of the phason wall of the strip and carries no distortions but only tile mismatches. The motion of the dislocation is therefore governed by two mechanisms: the first one, on the head, is driven by the usual elastic interactions with stress fields, whereas the second 
one, related to phasons, depends on the chemical ordering driving forces of the structure. The former mechanism induces possible fast motions but the latter one, relying on atomic diffusion, can have a much longer relaxation time depending on temperature. This suggests a scenario of dislocations being immobile at low temperature, having a slow motion and trailing a more or less extended phason wall at intermediate temperatures and eventually an easy viscous motion at higher temperatures as will be discussed next.

\subsection{Glide and climb in quasicrystals}

Formally the glide manifold associated to a dislocation is defined by the union of the dislocation line $V$ and the Burgers vector $\vec{B}$. As in ordinary crystals, a motion of the dislocation line in the glide manifold is conservative in the sense that it generates no long distance mass transport. In the icosahedral case, it is a generic $5 D$-space that decomposes into a $3 D$-space parallel to $\mathrm{E}_{\perp}$ plus a $2 D$-manifold in $\mathrm{E}_{\|}$that is the actual glide plane if the dislocation line is a straigh line. If this straight line is along a quasicrystalline direction, then the generic glide $5 D$-manifold contains a $3 D$-sublattice of $\Lambda$ defined by the $2 D$-sublattice of $\Lambda$ contained in $V$ and the Burgers vector $\vec{B}$ itself. The remaining $2 D$-subspace is spanned by two directions that are irrationally oriented with respect to $\Lambda$ and embedded in $E_{\perp}$.

Climb is defined as a motion direction of the dislocation line that has a non zero component outside the glide manifold. For example, this is the case for a dislocation moving along the direction $\vec{b}_{\|} \wedge \vec{v}_{\|}$in $\mathrm{E}_{\|}$which corresponds to pure climb motion like in ordinary crystals. It is a non-conservative motion that requires atomic propagation to long distance.

Because the motion of a perfect dislocation requires a re-tiling around the dislocation line, we can analyze it as a two-step process: a (pure phonon) motion characterized by $\vec{b}_{\|}$followed by the chemical reordering (pure phason) and re-tiling characterized by $\vec{b}_{\perp}$.

The question arises of what direction of motion, say $\vec{d}_{\|}$, would be optimum for the sole phonon field propagation irrespective of the phason field reconstruction.

We first notice atoms in quasicrystals distribute along (quasicrystalline) rows and planes as in crystals. Therefore, we may expect quasilattice friction to play a role with the existence of quasiperiodically spaced Peierls valleys along which dislocation lines would be preferencially oriented. For the same reasons, we assume that the direction of motion to be a quasicrystallographic direction, i.e. $\vec{d}_{\|}$can be considered, irrespective of a length factor, as the projection of a 
$6 D$ node of $\Lambda$ which, in turn, has a single image $\vec{d}_{\perp}$ in $\mathrm{E}_{\perp}$. We assume the dislocation line $\vec{v}_{\|}$to also be a straight line aligned along another quasicrystallographic direction not collinear to $\vec{d}_{\|}$where, as previously, $\vec{v}_{\|}$is the projection of a $6 D$-lattice node with image $\vec{v}_{\perp}$ in $\mathrm{E}_{\perp}$.

The surface swept by the dislocation during the motion is a $5 D$ space generated by the dislocation line $V$ (a $4 D$-manifold) plus the direction of motion $\vec{d}_{\|}$. This $5 D$-space, say $S$, contains a $4 D$-sublattice of $\Lambda$ embedded in the $4 D$-subspace, say $\Sigma$, generated by $\left(\vec{v}_{\|}, \vec{v}_{\perp}, \vec{d}_{\|}, \vec{d}_{\perp}\right)$. The remaining dimension corresponds to a direction $\Delta$ that is necessarily in $\mathrm{E}_{\perp}-$ since $\Sigma$ contains a $2 D$-plane only $\left(\vec{v}_{\perp}, \vec{d}_{\perp}\right)$ of the $3 D$-space $\mathrm{E}_{\perp}$ - and therefore irrationally oriented with respect to $\Lambda$. For the displacement by $\vec{b}_{\|}$of the matter during the dislocation motion to respect the tiling matching with no overlaps and empty spaces, the $4 D$-sublattice embedded in $S$, that represents the part of the tiling vertices that form the matches on the $\Sigma$ surface, must be kept invariant up to the global translation $\vec{b}_{\|}$in $\mathrm{E}_{\|}$.

This is achieved if $\vec{b}_{\perp}$ has a zero component in $\Sigma$ and thus is aligned along $\Delta$. This induces $\vec{b}_{\perp} \perp \vec{d}_{\perp}$ and $\vec{b}_{\perp} \perp \vec{v}_{\perp}$. Because for any two vectors $\vec{A}$ and $\vec{B}$ of $\Lambda$, a zero scalar product of their projections in either the spaces $\mathrm{E}_{\|}$or $\mathrm{E}_{\perp}$ implies the same zero value for the scalar product of the projections in the other space:

$$
\vec{A}, \vec{B} \in \Lambda, \quad \vec{a}_{\|} \cdot \vec{b}_{\|}=0 \leftrightarrow \vec{a}_{\perp} \cdot \vec{b}_{\perp}=0
$$

so that $\vec{b}_{\|} \perp \vec{d}_{\|}$and $\vec{b}_{\|} \perp \vec{v}_{\|}$. Hence, we can conclude that the dislocation motion that keeps the internal tiling coherent - with no empty spaces or overlapps in the trace of motion - is obtained for pure edge dislocation $\left(\vec{b}_{\|} \perp \vec{v}_{\|}\right)$moving in a pure climb fashion $\left(\vec{b}_{\|} \perp \vec{d}_{\|}\right)$.

This problem of glide versus climb in the dislocation motion in $i$ AlPdMn has been the object of interesting debates in these last years. Most of the early heuristic models of dislocation motion in $i$-AlPdMn where primarily focused on glide mechanisms [18, 19]. Due to the large dependence of mechanical properties on temperature, they aimed at describing macroscopic parameters in the framework of thermally activated dislocation motion. The thermally activated events were assumed to correspond to the overcoming of atomic clusters or of a quasi-periodic Peierls barrier. They accounted for activation parameters satisfactorily at low temperatures and high stresses. However, since the year 2000, numerous experimental evidences for climb have been found in as cast $i$-AlPdMn [21, 22, 23], deformed at low [24] and at high temperatures [25]. Nowadays, although it seems that climb has been accepted as the major mechanism of plastic deformation of $i$-AlPdMn at high temperature. 
Figure 2 shows a dislocation $d$ moving at $750{ }^{\circ} \mathrm{C}$ in $i$-AlPdMn. Its velocity is around $40 \mathrm{~nm} / \mathrm{s}$ and its motion is viscous (Fig. 2a-c). During its motion, an elastic distortion has been left at the sample surfaces giving rise to two straight and dark traces labeled tr.P. This indicates that the plane of motion of the dislocation is inclined with respect to the foil plane. From the variation of its apparent width and trace direction with specimen tilt, the plane of motion has been found to be the $(0,1,0, \overline{1}, 0,0)$ two-fold plane $P_{2}$, of direction $[1 / 1,0 / \overline{1}, 1 / 0]$ (see stereographic projection on Fig. 2j). During its motion, the dislocation remains straight along the 2 -fold direction labeled $u$. According to the invisibility criterion $\vec{G} \cdot \vec{B}=0$, the two strong extinctions in $\vec{g}_{2 a}$ and $\vec{g}_{5 a}$ (Fig. 2d and e) lead to a Burgers vector $\vec{b}_{\| 1}$ perpendicular to $\vec{g}_{2 a}$ and $\vec{g}_{5 a}$, i.e. parallel to the direction $[1 / 1,0 / \overline{1}, 1 / 0]$ (Fig. $2 \mathrm{j}$ ). This Burgers vector is perpendicular to the plane of motion. This proves that the dislocation has moved by climb. Single contrast conditions $(\vec{G} \cdot \vec{B}=1)$ in $\vec{g}_{2 b}$ and $\vec{g}_{5 a}$ (Fig. 2f and g) and double contrast conditions $(\vec{G} \cdot \vec{B}=2)$ in $\tau \vec{g}_{2 c}$ and $\tau \vec{g}_{2 d}$ (Fig. $2 \mathrm{~h}$ and i) finally lead to $\vec{B}_{1}=(0,1,0, \overline{1}, \overline{1}, \overline{1})$. Its component in the physical space is $\vec{b}_{1 \|}=[1 / 0,1 / \overline{1}, 2 / \overline{1}]$ of length $0.297 \mathrm{~nm}$. The same dislocation has already been found in samples deformed at lower temperature (see [24]). From this and other experiments $[25,26]$, the following conclusions can be drawn:

- Dislocations move by climb in 2-, 3- and 5-fold planes. Glide is at least 1000 times slower than climb in similar conditions.

- Dislocations are straight along dense directions (mostly 2-fold directions). This implies the existence of Peierls valleys along which dislocations are more stable. The rectilinear aspect of dislocations during their motion suggests, by analogy with dislocation motion in covalent and BCC crystals, that dislocation velocity is controlled by a difficult jog pair nucleation over the Peierls valleys followed by their easy diffusion along the line like in BCC structures.

\section{Reconstructed electron density around dislocations}

As perfect quasicrystal, we use a polyhedral model recently proposed by Quiquandon et al. [15] for $i$-AlPdMn . The model is a chemical decoration of a skeleton of interconnected atomic clusters of two kinds, $B$ (Bergman) and $M$ (Mackay) (see $[16,17])$. The chosen decoration leads to a remarkably reduced number of local chemical 
configurations: a single major chemical configuration for each of the $M\left(M^{\prime}\right)$-type clusters and only four for the $B$-type cluster. It fits the experimental optimal composition $\mathrm{Al}_{70.35} \mathrm{Pd}_{21.36} \mathrm{Mn}_{8.29}$ and has reasonable reliability factors for both X-rays and neutrons diffraction spectra. Details on the model are to be found elsewhere [15].

To visualize the atomic displacements and rearrangements around a quasicrystalline dislocation, an image reconstruction is performed by Fourier transforming the scattering structure factors $F(\vec{Q})$ of the model multiplied by the phase factors due to the $6 D$-displacement associated with the dislocation:

$$
\varrho(\vec{R})=\left|\sum_{k} F\left(\vec{Q}_{k}\right) e^{2 i \pi\left(\vec{Q}_{k} \cdot\left(\vec{R}+\vec{U}\left(\vec{r}_{\|}\right)\right)\right)}\right|^{2}
$$

where $\vec{Q}_{k}$ are the $6 D$-wavevectors of the reciprocal $6 D$-lattice and $\vec{U}(\vec{r} \|)$ the total $6 D$ displacement field. This procedure has been first used by Devaud-Rzepski et al. [27] in the early 90's on simple quasilattice models and developed in much details by Yang et al. [28] on more realistic spherical atomic models. The interest of that kind of calculations is that images can be computed in which the two fields in $\mathrm{E}_{\|}$and $\mathrm{E}_{\perp}$ can be separated thus allowing to separate visually the phonon field from the phason field in $\mathrm{E}_{\|}$. The present calculations do not differ basically from the previous cited ones except that the structural atomic model is slightly more elaborated and the calculations are made with a large number of Fourier terms of the electron density, enough for localizing individual atoms.

The displacement phonon field in $\mathrm{E}_{\|}$is approximated by that of an ideal isotropic elastic medium. For straight dislocation lines, it is independent of the location of the running point with respect to its component along the line $\vec{v}_{\|}$and can thus be expressed as a function of the two cylindrical variables defined in the plane perpendicular to the dislocation line and passing through the running point. With the notations of Fig. 3 we use the standard formula:

$$
2 \pi \vec{u}_{\|}(\rho, \theta)=\vec{b}_{\|} \theta+\frac{\vec{b}_{\|, e} \sin 2 \theta+\left(\vec{b}_{\|} \wedge \vec{v}_{\|}\right)(2(1-2 \nu) \log \rho+\cos 2 \theta)}{4(1-\nu)}
$$

where $\vec{b}_{\|, e}$ is the edge part of $\vec{b}_{\|}$:

$$
\vec{b}_{\|, e}=\vec{b}_{\|}-\left(\vec{b}_{\|} \cdot \vec{v}_{\|}\right) \vec{v}_{\|}
$$

and $\rho=\sqrt{x^{2}+y^{2}}$ and $\theta=\arctan y / x$ with:

$$
x=\vec{r}_{\|} \cdot \vec{b}_{\|, e} /\left|\vec{b}_{\|, e}\right|, \quad y=\vec{r}_{\|} \cdot\left(\vec{b}_{\|} \wedge \vec{v}_{\|}\right) /\left|\vec{b}_{\|} \wedge \vec{v}_{\|}\right| .
$$


For the phason displacement field (in $\mathrm{E}_{\perp}$ ), we use here the simplest pure screw model $^{2}$ :

$$
2 \pi \vec{u}_{\perp}\left(\vec{r}_{\|}\right)=\vec{b}_{\perp} \theta
$$

The effect of each of the displacement fields in $\mathrm{E}_{\|}$and $\mathrm{E}_{\perp}$ are exemplified on Fig. 4. The Fourier reconstruction of Fig. 4a has been computed in including only the phase shift of the parallel space contribution $\vec{b}_{\|}$(phonon field). A strong misfit of the atomic clusters is observed at the level of the Volterra (arbitrary) cut surface shown as a light gray line, that generates a phason wall along the line. This phason wall is very similar to a classical stacking fault boundary with the exception that the components of the fault vector have the form $(n+m \tau) / d$ where $n, m$ and $d$ are integers. Close to the dislocation core located at the center of the image, there exist a significant local deformation of the cluster shapes due to the elastic field of the dislocation. This, of course, is physically irrelevant since in that core region, linear elasticity is no longer valid.

The Fourier reconstruction of Fig. 4b uses the same model but has been computed with the perpendicular component $\vec{b}_{\perp}$ only, the phason field. One observes again a strong misfit at the level of the Volterra cut that shows, like in the previous case and as expected, that the closure of the $6 D$ hyper-crystal is not achieved by the sole $\vec{b}_{\perp}$ component. As this displacement field extends only in $\mathrm{E}_{\perp}$ and in contrast to the previous case, there are no changes in the shape of the atomic clusters that are everywhere identical to those of the perfect quasicrystal. The difference here is in the way they are connected together.

The image of a perfect dislocation is fully recovered on picture (c) when both components $\vec{b}_{\|}$and $\vec{b}_{\perp}$ are taken into account. In that case, the trace of the Volterra cut surface disappears because of the proper restacking issued from the perpendicular translation and the deformation spread out all over the atomic clusters as expected by the generated elastic field.

Figure 5 shows a high resolution reconstructed image of the electron density of the $i$-AlPdMn projected along the 3 basic high symmetry directions around a typical other dislocations of Burgers vector $\vec{B}=(0,0,1,0,0, \overline{1})$ of parallel component $\vec{b}_{\|}=[0 / 0,2 / 0,0 / 0]$ with $\left|b_{\|}\right|=0.4795 \mathrm{~nm}$ and $\left|b_{\perp}\right|=0.7759 \mathrm{~nm}$.

The dislocation seen on Fig. 6 are partial dislocations of Burgers vectors $\vec{B}_{1}=(\overline{1}, 1,1,1,1, \overline{1}) / 2$ and $\vec{B}_{2}=(1,0,0,0,0,0)$; they are the

\footnotetext{
${ }^{2}$ There are a priori no basic physical reasons for choosing this particular law except that it can be reasonably assumed that the phason field spreads out isotropically around the dislocation line.
} 
termination of fault planes with the same fault vectors drawn in light grey on the picture. These two faults correspond to the well-known planar defects with $\pi$ fringes as antiphase boundaries in ordinary crystals.

\section{Conclusion}

Dislocations are defined in quasicrystals in much the same way as they are in ordinary crystals. Because of the additional dimensions needed to recover periodicity, the Burgers vector $\vec{B}$ of a perfect dislocation is a translation of the high dimensional lattice, and decomposes into two irreducible components, $\vec{b}_{\|}$and $\vec{b}_{\perp}$, corresponding respectively to the phonon field in $\mathrm{E}_{\|}$(standard elastic deformation) and the phason field developing in $\mathrm{E}_{\perp}$ (configurational thermodynamical energy). These two components are the physical bases of the dislocation motion mechanism that can be described as difficult jog pair nucleation over quasiperiodic Peierls valleys followed by a relatively easy diffusion along the line at high temperatures.

The geometrical framework of quasicrystalline dislocation description in high dimension spaces is presently well established. The unerstanding of the underlying physics gouverning their interactions and their modes of motion is at its beginning. This research field should open new original ways for studying the high temperature plastic behaviour of complex intermetallic phases with large unit cells which share many geometrical properties with quasicrystals.

\section{References}

[1] D. Shechtman, I. Blech, D. Gratias, and J. W. Cahn.Phys. Rev. Lett., 53:1951-1954 (1984).

[2] D. Shechtman and I. Blech.Met. Trans., 16A:1005-1012 (1985).

[3] M. Wollgarten, Z. Zhang and K. Urban, Phil Mag Lett, 65(1) 1-6 (1992).

[4] M. Wollgarten, M. Beyss, K. Urban, H. Liebertz and U. Kocster, Phys. Rev. Lett., 71, 543 (1993).

[5] T. C. Lubensky, S. Ramaswamy, and J. Toner. Phys. Rev. B, 33:7715-7719 (1986).

[6] J. E. S. Socolar, T. C. Lubensky, and P. J. Steinhardt. Phys. Rev. $B$, 34:3345-3360 (1986). 
[7] M. Kléman, M. in Proceedings of the ILL/Codest Conference (Grenoble) Edited by C. Janot and J. M. Dubois, World Scientific(Singapor), 318-326 (1988).

[8] M. Kleman and K. Sommers Acta Met. Mat. 39, 287 (1991).

[9] M. Duneau and A. Katz, Phys. Rev. Lett. 54, 2688-2691 (1985); A. Katz and M. Duneau, J. Phys. France 47, 181-196 (1986).

[10] P. A. Kalugin, A. Y. Kitayev and L. S. Levitov, JETP Lett. 41, 145 (1985);ibid. J. Phys. Lett. France46, L601 (1985).

[11] V. Elser, Acta Cryst. A 42, 36 (1986).

[12] P. Bak, Phys. Rev. B 32, 5764 (1985); ibid. Scripta Met. 20, 1199-1204 (1986);ibid. Physica B\&C 136, 296-300 (1986).

[13] J. E. S. Socolar, P. J. Steinhardt and D. Levine, Phys. Rev. B 32 5547 (1985)

[14] J. W. Cahn, D. Shechtman and D. Gratias, J. Mater. Res, 1, 13 (1986) .

[15] M. Quiquandon, J.-T. Beauchesne and D. Gratias, Prototypical simple atomic structure of $i-A l P d M n$, in preparation.

[16] A. Katz and D. Gratias ,J. Non-Crystalline Solids 153-154, 187195 (1993).

[17] D. Gratias, F. Puyraimond, M. Quiquandon and A. Katz, Phys. Rev. B 63 024202-1-15 (2000).

[18] M. Feueurbacher, C. Metzmacher, M. Wollgarten, B. Baufeld, M. Bartsch, U. Messerschmidt and K. Urban, K.,Mater. Sci. Engng, A233, 103 (1997).

[19] S. Takeuchi, R. Tamura and E. Kabutoya, Phil. Mag. A82, 379 (2002) ; see also S. Takeuchi, Mater. Res. Soc. Symp. Proc., 553, 283 (1999) .

[20] U. Koschella, PhD dissertation Phason-elastische Energie in dekagonalen Quasikristallen, Stuttgart University (2005).

[21] D. Caillard, G. Vanderschaeve, L. Bresson and D. Gratias, Phil. Mag. A80, 237 (2000).

[22] D. Caillard, J. P. Morniroli, G. Vanderschaeve, L. Bresson and D. Gratias, Eur. Phys. J. Appl. Phys.,20, 3 (2002).

[23] D. Caillard, C. Roucau, L. Bresson and D. Gratias, Acta Mater., 50, 4499 (2002).

[24] F. Mompiou, L. Bresson, P. Cordier and D. Caillard,Phil. Mag., 83, 3133 (2003). 
[25] F. Mompiou, M. Feuerbacher and D. Caillard, Phil. Mag., 84, 2777-2792 (2004) .

[26] F. Mompiou and D. Caillard, Phil. Mag. Lett., 84, 555 (2004).

[27] J. Devaud-Rzepsky, M. Cornier-Quiquandon and D. Gratias in Quasicrystals and incommensurate structures in condensed matter, edited by M. J. Yakaman, D. Romen, V. Castano, A. Gomez, (Singapore: World Scientific) p.498 (1990).

[28] W. Yang, M. Feuerbacher, N. Tamura, D. H. Ding, R. Wang and K. Urban, Phil. Mag. A, 77, 1481 (1998). 
Figure 1: Example of an hypothetical dislocation of Burgers vector $B=$ $(0,0,1,0,0,-1)$ (indexing scheme of [14]) seen end on in a 5 -fold plane of an ideal atomic model of $i$-AlPdMn. This numerical reconstruction is computed using formula 3 including 141 beams of the 5 -fold plane based on the structural model of $i$-AlPdMn . As in ordinary crystals, this "edge" dislocation is clearly visible at glancing angle as a discontinuity of atomic "planes".

Figure 2: Dislocation motion in the 2-fold plane P2 (a-c). Note the growth of the precipitate PR. The extinction condition (d-e), single (f-g) and double contrast conditions (h-i) lead to the burgers vector noted $b_{1 \|}$ on the stereographic projection $(\mathrm{j})$. The experimental determination of the Burgers vector together with the determination of the plane of motion of the dislocation shows that the motion process has a pure climb character.

Figure 3: Geometrical variables in $\mathrm{E}_{\|}$used to calculate the displacement field of a dislocation of Burgers vector $\vec{b}$ with a (straight) dislocation line along the unit vector $\vec{v}$. The local reference frame is defined by: $z$ is along the dislocation line, $x$ is the direction of the edge part of the dislocation, $\vec{b}_{e}-$ and $\vec{b}_{s}$ is its screw part - and $y$ is the direction perpendicular to $z$ and $x$. The running point $\vec{r}$ is in the plane $(x, y)$ and has cylindrical coordinates $(\rho, \theta)$ where the origin of the angle $\theta$ is taken along the positive $x$ axis.

Figure 4: The various components of the phase shift induced by a perfect dislocation $\vec{B}=\vec{b}_{\|}+\vec{b}_{\perp}=(\overline{1}, 1,0,1,1,0)$ with $\vec{b}_{\|}=[\overline{2} / 2,0 / 2,0 / 0]$ and $\left|b_{\|}\right|=0.296381 \mathrm{~nm} ;\left|b_{\perp}\right|=1.25549 \mathrm{~nm}$ of dislocation line $\vec{u}_{\|}=[0 / 0,0 / 0,1 / 0]$ in the $i$-AlPdMn model built with B-clusters (pentagons) and M-clusters (decagons). The dislocation is seen end-on in the 5-fold plane perpendicular to $(0,1, \tau)$. Structural Fourier reconstruction: (a) phonon field: including only the $\vec{b}_{\|}$component, (b) phason field: including only the $\vec{b}_{\perp}$ and (c) using both components. The light gray line is the trace of the Volterra cut surface: it appears as a line discontinuity on (a) and (b) reconstructions but, of course disappears in the full reconstruction (c).

Figure 5: High resolution calculated electron density of the $i$-AlPdMn model around a dislocation of Burgers vector $\vec{B}=(\overline{1}, 1,0,1,1,0)$ for from left to right the phonon $(\mathrm{a}, \mathrm{d}, \mathrm{g})$, phasons $(\mathrm{b}, \mathrm{e}, \mathrm{h})$ and total $(\mathrm{c}, \mathrm{f}, \mathrm{i})$ displacement fields projected along the 5 - fold $(\mathrm{a}, \mathrm{b}, \mathrm{c})$, the 2 -fold $(\mathrm{d}, \mathrm{e}, \mathrm{f})$ and 3 -fold $(\mathrm{g}, \mathrm{h}, \mathrm{i})$ directions. 
Figure 6: Reconstructed electron density of the $i$-AlPdMn model for partial dislocations of Burgers vectors $(\overline{1}, 1,1,1,1, \overline{1}) / 2$ and $(1,0,0,0,0,0)$ seen perpendicular to a 2 -fold direction. 